Urologe $2021 \cdot 60: 1025-1034$

https://doi.org/10.1007/s00120-021-01608-3

Angenommen: 8. Juli 2021

Online publiziert: 14. Juli 2021

( $)$ Springer Medizin Verlag $\mathrm{GmbH}$, ein Teil von Springer Nature 2021

\section{Weiterbildungs- und Arbeitsbedingungen urologischer Ärztinnen und Ärzte in Weiterbildung in Deutschland}

\author{
Ergebnisse der zweiten Weiterbildungsumfrage der German \\ Society of Residents in Urology e. V. (GeSRU) von 2020
}

\author{
H. Arnold $\cdot$ M. Fassbach ${ }^{2} \cdot$ A. Mattigk ${ }^{3} \cdot$ V. Zehe ${ }^{3} \cdot$ A. Beck B F. Wundrack $^{5} \cdot$ L. Bellut ${ }^{6} \cdot$ \\ J. König ${ }^{7} \cdot$ C. Siech ${ }^{8}$ \\ 'Facharztpraxis, Urologie Dr. Hannah Arnold, Gesundheitszentrum Senden, Senden, Deutschland; ${ }^{2}$ Klinik \\ für Urologie, urologische Onkologie und Kinderurologie, Helios Klinikum Duisburg, Duisburg, Deutschland; \\ ${ }^{3}$ Klinik für Urologie und Kinderurologie, Universitätsklinikum Ulm, Ulm, Deutschland; ${ }^{4}$ Klinik für Urologie, \\ Katholisches Marienkrankenhaus Hamburg, Hamburg, Deutschland; ${ }^{5}$ Klinik für Urologie, Helios Klinikum \\ Berlin-Buch, Berlin-Buch, Deutschland; ${ }^{6}$ Klinik für Urologie und Kinderurologie, Universitätsklinikum

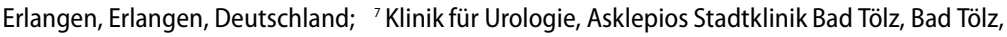 \\ Deutschland; ${ }^{8}$ Klinik für Urologie, Universitätsklinikum Frankfurt, Frankfurt am Main, Deutschland
}

\section{Zusatzmaterial online}

Die Online-Version dieses Beitrags (https:// doi.org/10.1007/s00120-021-01608-3) enthält die dieser Arbeit zugrunde liegenden Fragebögen der Weitbildungsumfragen 2015 und 2020.

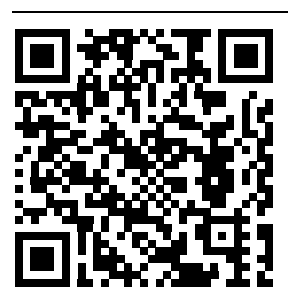

QR-Code scannen \& Beitrag online lesen

\section{Zusammenfassung}

Hintergrund: Die Zeit der ärztlichen Weiterbildung markiert den Karrierebeginn junger Urolog:innen und ist damit ein Grundstein für die zukünftige Krankenversorgung. 2015 führte die German Society of Residents in Urology e. V. (GeSRU) erstmals eine Befragung unter den Ärzt:innen in urologischer Weiterbildung durch und beschrieb den Status quo deren Weiterbildung. Mit dieser Folgeuntersuchung soll ein aktualisiertes Abbild der Weiterbildungs- und Arbeitsbedingungen junger Urolog:innen erstellt und eine Verlaufsbeurteilung ermöglicht werden.

Methoden: Die GeSRU führte 2020 eine onlinebasierte Befragung aller Ärzt:innen in urologischer Weiterbildung durch. Dafür wurde der Fragebogen von 2015 ausgebaut. Es wurde erneut das Modell der beruflichen Gratifikationskrise eingesetzt.

Ergebnisse: Insgesamt wurden 332 Fragebögen ausgewertet. Im Vergleich zu 2015 ergaben sich keine wesentlichen Änderungen. Der Arbeitsalltag stellt sich weiterhin stark verdichtet dar. Zudem ist der Einfluss ökonomischer Erwägungen auf die Teilnehmenden weiterhin deutlich spürbar. Familien- und forschungsfreundliche Arbeitsbedingungen fehlen weitgehend. $35 \%$ der Befragten ziehen hieraus berufliche Konsequenzen. Die psychosoziale Belastung ist sehr hoch und stellt ein Risiko für die Gesundheit der Ärzt:innen selbst sowie für die Behandlungsqualität ihrer Patient:innen dar.

Schlussfolgerung: Auf Basis dieser Befragung bestehen weiterhin systemrelevante Belastungen im Arbeitsleben junger Ärzt:innen in urologischer Weiterbildung, die Anpassungen der Arbeits- und Weiterbildungsbedingungen erfordern. Ein strukturiertes, transparentes Weiterbildungscurriculum, Vergütung von und Zeit für gute Weiterbildung und Modelle zur Vereinbarkeit von Forschung bzw. Familie und Beruf sollten etabliert werden.

\section{Schlüsselwörter}

Urologische Weiterbildung · Arbeitsbelastung · Ökonomisierung · Gratifikationskrise · Operative Ausbildung 


\section{Einleitung}

Die ärztliche Weiterbildung in Deutschland verändert sich. Mit der Verabschiedung der neuen Musterweiterbildungsordnung (MWBO) 2018 wurde durch den Deutschen Ärztetag der Weg zu einer kompetenzorientierten Weiterbildung gebahnt [5]. Darauf aufbauend hat die Deutsche Gesellschaft für Urologie (DGU) in Kooperation mit der German Society of Residents in Urology e.V. (GeSRU) sowie dem Berufsverband Deutscher Urologen (BvDU) das "Weiterbildungscurriculum Urologie" (WECU) entwickelt. Es soll strukturiert und vergleichbar weitergebildet werden, um die Qualität der Patientenversorgung zu steigern und das Fach Urologie attraktiver für den Nachwuchs zu gestalten [25].

In den nächsten 10 Jahren wird der urologische Versorgungsbedarf um $20 \%$ steigen [34]. Zudem werden in 15 Jahren $74 \%$ der aktuell praktizierenden Urolog:innen berentet sein [11]. Ein verändertes ärztliches Selbstverständnis und Vorgaben des Arbeitszeitgesetzes verändern auch die urologischen Arbeitsabläufe in den Kliniken und Praxen. Eltern nehmen mehr Auszeiten für die Kindererziehung und Forschende wünschen geschützte Zeit für wissenschaftliches Arbeiten $[2,16]$.

Um die Weiterbildungs- und Arbeitsbedingungen urologischer Ärzt:innen in Deutschland zu diskutieren, hat die GeSRU bereits 2015 eine erste große Online-Befragung durchgeführt. Hier zeigten sich die Teilnehmenden trotz einer hohen psychosozialen Arbeitsbelastung insgesamt mit inrer beruflichen Situation zufrieden. Sie äußerten aber deutliches Verbesserungspotential bei der Supervision sowie der Vereinbarkeit von Beruf, Familie und Forschung [3].

Ziel dieser Studie ist im Auftrag der AG Junge Urologen der DGU und der GeSRU die erneute systematische Erhebung eines Meinungsbildes über die Qualität und Rahmenbedingungen der urologischen Weiterbildung in Deutschland im Jahr 2020, um Chancen und Herausforderungen zu analysieren sowie Lösungsansätze und Perspektiven zu formulieren. Zudem soll die Lage vor der flächendeckenden Anwendung des WECU und der neuen kompetenzorientierten $\mathrm{MWBO}$, welche im Juli 2020 in Kraft trat, dargestellt, sowie
Veränderungen im Vergleich zur Vorumfrage von 2015 aufgezeigt werden, um die Zukunft der Urologie gemeinsam mit dem urologischen Nachwuchs zu gestalten.

\section{Methoden}

\section{Studienpopulation}

Zielgruppe der anonymisierten, digitalen Umfrage waren alle Ärzt:innen in Weiterbildung (ÄiW) für Urologie in Deutschland. Der Befragungszeitraum umfasste Juli bis Dezember 2020. Der Aufruf zur Teilnahme erfolgte über den Newsletter der GeSRU an alle eingetragenen Mitglieder, die öffentlich zugängliche GeSRU-Homepage sowie die Social-media-Kanäle der GeSRU. Erinnerungen wurden alle 2 Wochen verschickt. Zusätzlich erfolgte im Oktober die Bewerbung der Teilnahme an der Umfrage in diversen Fachzeitschriften sowie im Dezember der Versand des Umfragelinks über den E-Mail-Verteiler der Leitenden Ärzt:innen der DGU. Die Umfrage wurde über SurveyMonkey ${ }^{\circledR}$ (Survey Monkey Inc., San Mateo, CA, USA) durchgeführt.

\section{Fragebogen}

Die Befragung umfasste insgesamt 82 Fragen mit einer minimal bzw. maximal möglichen Anzahl von 68 bzw. 77 Fragenjenach Folgeantwortlogik. Die Umfrage wurde in 8 Abschnitte unterteilt:

1. Arbeitsbedingungen im Berufsalltag (7-9 Fragen),

2. ärztliche Fort- und Weiterbildung (8 Fragen),

3. operative Ausbildung (13 Fragen),

4. Vereinbarkeit von Beruf und Familie (9 Fragen),

5. Einfluss ökonomischer Erwägungen auf ärztliches Handeln (7 Fragen),

6. Vereinbarkeit von klinischem Arbeitsalltag mit Forschung und Wissenschaft (3-7 Fragen),

7. arbeiten in der Schwangerschaft (0-4 Fragen)

8. Basisdaten (18 Fragen).

Struktur und Inhalt des Fragebogens orientieren sich zum Großteil an der Vorbefragung von 2015 sowie an Befragungen von ÄiW im Fachbereich Innere Medizin $[29,31]$, um eine inter- sowie intradiszi- plinäre Vergleichbarkeit der Erhebung zu sichern.

Erneut wurde wieder die Kurzfassung des validierten Fragebogens zum Modell der beruflichen Gratifikationskrisen verwendet (2 Fragen mit jeweils 8 Unterfragen, "Effort-reward-imbalance“[ERI]Fragebogen mit 4-Punkte-Likert-Skala). Dabei handelt es sich um ein Selbstbeurteilungsverfahren zur Erfassung von arbeits- und berufsbezogenen Stresserfahrungen. Das Modell geht davon aus, dass ein Missverhältnis von hohen geleisteten arbeits- bzw. berufsbezogenen Verausgabungen einerseits und niedriger Gratifikation (Belohnungen in Form von Bezahlung, Wertschätzung, beruflichem Aufstieg, Arbeitsplatzsicherheit) andererseits zu Stresserleben und gesundheitlichen Beeinträchtigungen führt. Die Gratifikationskrise fällt umso stärker aus, je höher die geleistete Verausgabung im Verhältnis zu den erfahrenen Gratifikationen ist. Außerdem wird im Modell angenommen, dass ein höheres Belastungs- bzw. Krankheitsrisiko dann besteht, wenn eine hohe berufliche Verausgabungsneigung vorliegt.

Zur Vergleichbarkeit der Ergebnisse erfolgte die Adjustierung der drei Subskalen für den Umfang des Fragebogens und die Skalierung. Der aus den adjustierten Werten von Verausgabung und Belohnung gebildete Quotient (ER-Ratio) stellt eine quantitative Abschätzung des Ausmaßes beruflicher Gratifikationskrisen dar.

Neuer Themenkomplex der Umfrage bildete der Abschnitt "Arbeiten in der Schwangerschaft". Darüber hinaus wurden die Bereiche "Arbeitsbedingungen im Berufsalltag" um drei Fragen und "Vereinbarkeit von Beruf und Familie" um eine Frage erweitert sowie „Basisdaten" um zwei Fragen reduziert und eine Frage ergänzt. Die restlichen Bereiche blieben im Vergleich zur Befragung 2015 unverändert.

\section{Statistik}

Die statistische Auswertung wurde mit SPSS 27.0 (IBM, New York, USA) durchgeführt. Als parametrische Verfahren wurden zum Vergleich von 2 Gruppen der t-Test für unabhängige Stichproben (95\%-Konfidenzintervall [KI]) oder bei $\geq 2$ Gruppen 
Tab. 1 Basisdaten der deutschlandweiten Befragungen von Ärzt:innen in Weiterbildung für Urologie 2015 und $2020 \mathrm{im}$ Vergleich

\begin{tabular}{|c|c|c|c|}
\hline \multicolumn{2}{|l|}{ Merkmal } & \multicolumn{2}{|c|}{ Jahr der Befragung } \\
\hline & & 2020 & 2015 \\
\hline Teilnehmende gesamt & - & 332 & 472 \\
\hline Geschlecht (w. vs. m [\%]) & - & 53 vs. 47 & 55 vs. 45 \\
\hline Alter (Jahre) $)^{\mathrm{a}}$ & - & $31 \pm 3,0$ & $31 \pm 4,0$ \\
\hline Weiterbildungsjahr (WBJ) ${ }^{\mathrm{a}}$ & - & $3,4 \pm 1,6$ & $3,5 \pm 1,7$ \\
\hline Erwachsene im Haushalt (\%) & 1 vs. $\geq 2$ & 31 vs. 69 & 39 vs. 61 \\
\hline Kinder im Haushalt (\%) & 0 vs. $\geq 1$ & 72 vs. 28 & 72 vs. 28 \\
\hline Zeitliche Arbeitsweise (\%) & Voll- vs. Teilzeit & 92 vs. 8 & 90 vs. 10 \\
\hline $\begin{array}{l}\text { Trägerschaft des Arbeitgebers } \\
\text { (\%) }\end{array}$ & $\begin{array}{l}\text { Öffentlich vs. Freigemeinnützig } \\
\text { vs. privat }\end{array}$ & $\begin{array}{l}52 \text { vs. } 31 \text { vs. } \\
17\end{array}$ & $\begin{array}{l}60 \text { vs. } 25 \text { vs. } \\
15\end{array}$ \\
\hline Versorgungstyp (\%) & $\begin{array}{l}\text { Universitätsklinikum vs. Maxi- } \\
\text { malversorger vs. } \\
\text { Grund-, Regel- und Schwer- } \\
\text { punktversorger }\end{array}$ & $\begin{array}{l}28 \text { vs. } 28 \text { vs. } \\
41\end{array}$ & $\begin{array}{l}26 \text { vs. } 33 \text { vs. } \\
41\end{array}$ \\
\hline \multirow[t]{4}{*}{ Wochenarbeitsstunden (\%) } & $<40$ & 6 & 8 \\
\hline & $40-49$ & 25 & 23 \\
\hline & $50-59$ & 43 & 43 \\
\hline & $\geq 60$ & 25 & 26 \\
\hline \multirow{4}{*}{$\begin{array}{l}\text { Durchschnittliche Anzahl an } \\
\text { Diensten/Monat (\%) }\end{array}$} & Keine & 1 & - \\
\hline & $1-3$ & 15 & - \\
\hline & $4-6$ & 67 & - \\
\hline & $6-10$ & 17 & - \\
\hline \multirow{3}{*}{$\begin{array}{l}\text { Durchschnittliche Anzahl an } \\
\text { Arbeitstagen am Wochenen- } \\
\text { de/Monat (\%) }\end{array}$} & Keine & 1 & - \\
\hline & $1-3$ & 81 & - \\
\hline & $4-6$ & 18 & - \\
\hline
\end{tabular}

eine ANOVA mit Tukey als Post-hoc-Test verwendet. Als nicht-parametrische Verfahren kamen der Mann-Whitney-U- und der Kruskal-Wallis-Test zum Einsatz. Beobachtete und erwartete Verteilungen wurden durch Kreuztabellen verglichen und durch $x^{2}$-Test auf Signifikanz getestet. Ein $p$-Wert $<0,05$ wurde als statistisch signifikant gewertet.

Für den jeweiligen Test wurden zusätzlich etablierte Kennzahlen der Effektstärke angegeben: t-Test: Cohen's d (Effektgröße für Mittelwertunterschiede), ANOVA: $\eta^{2}$ (Anteil erklärter Varianz), MWU-test: $r$ (Effektgröße für Medianunterschiede), $X^{2}$ : Cramer-V (Zusammenhangsmaß).

\section{Ergebnisse}

\section{Basisdaten}

Insgesamt haben 332 ÄiW an der Umfrage teilgenommen. Eine bundesweite Gesamtanzahl der ÄiW für Urologie wird in offiziellen Statistiken nicht geführt. Basie- sind unentschieden und $24 \%$ sind eher oder sehr unzufrieden.

Als Gründe für die angegebene Unzufriedenheit wurden insbesondere ein zu hoher Anteil nichtmedizinischer Tätigkeiten im Berufsalltag (42\%), die Arbeitsverdichtung (41\%) und eine mangelnde Qualität der Weiterbildung (34\%) genannt. Außerdem wurden zu viele Arbeitsstunden pro Monat (29\%), eine fehlende Anleitung und/oder Supervision (23\%), ein zu großer Einfluss ökonomischer Faktoren auf fachlich-medizinische Entscheidungen (20\%), eine schlechte Vereinbarkeit von Familie und Beruf (19\%) und eine geringe Anerkennung für geleistete Arbeit (18\%) angegeben. Seltene Gründe waren ein schlechtes Arbeitsklima (6\%), zu steile Hierarchien (5\%) oder mangelnde Autonomie (3\%). Männliche Befragte geben im Vergleich zu weiblichen Befragten signifikant häufiger einen zu hohen Anteil nicht-medizinischer Tätigkeiten im Berufsalltag als Grund für ihre Unzufriedenheit an (51\% vs. $35 \%$; t-Test; $p<0,05)$.

Durch die Veränderungen im ärztlichen Arbeitsumfeld über die letzten 5 bis 10 Jahre sehen $78 \%$ die Qualität der Krankenversorgung gefährdet. Gründe hierfür stellen v. a. die Arbeitsverdichtung (87\%), eine Zunahme nichtärztlicher Tätigkeiten (75\%) und mangelnde ärztliche Fort- und Weiterbildung $(41 \%)$ dar.

Eine vollständige Dokumentation von Überstunden geschieht bei $61 \%$ der Befragten. Nur $39 \%$ erhalten eine vollständige Vergütung ihrer Überstunden, die bei $11 \%$ vollständig monetär, $34 \%$ anteilig monetär und in Freizeit und bei $43 \%$ ausschließlich durch Freizeitausgleich erfolgt. Hier ergeben sich keine signifikanten Unterschiede zwischen den drei Gruppen der Klinikträger.

Aufgrund ihrer Unzufriedenheit haben bereits 35\% der Befragten Konsequenzen gezogen, häufiger als noch 2015 (• Abb. 2). $80 \%$ haben aufgrund ihrer Unzufriedenheit zumindest erwogen ihre Arbeitssituation zu verändern.

\section{Ärztliche Fort- und Weiterbildung}

Rund $23 \%$ bekamen von ihrem Weiterbildenden eine Struktur für die Weiterbildung in Form eines Curriculums oder Weiterbildungsplans. Die Hälfte (51\%) rechnet da- 


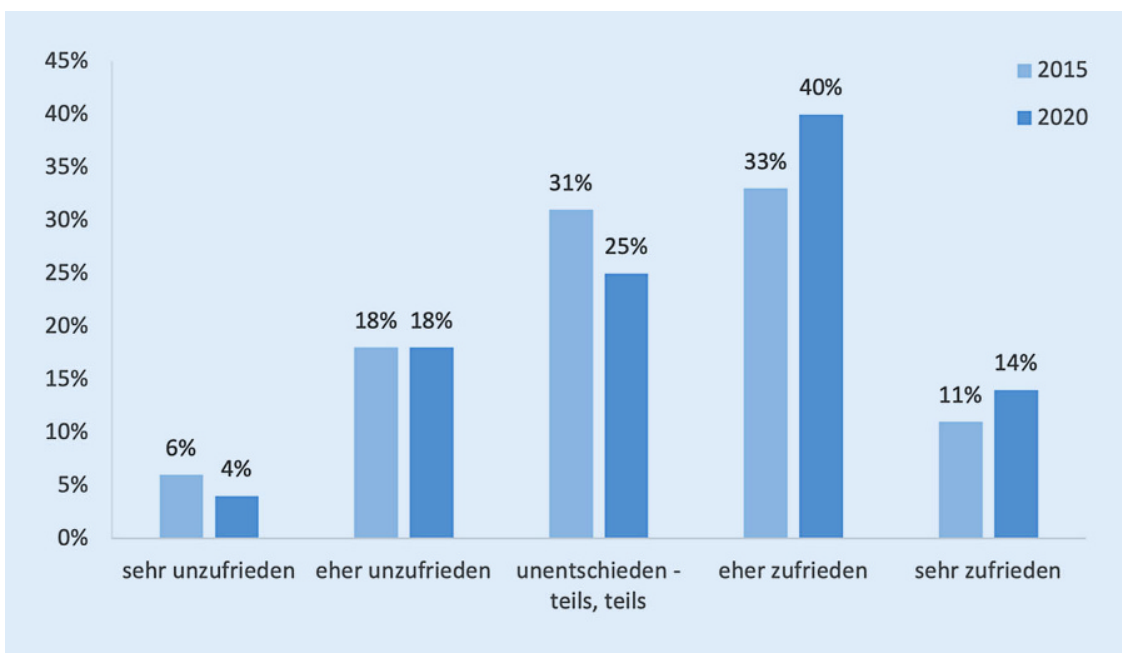

Abb. $1 \Delta$ Zufriedenheit von Ärzt:innen in Weiterbildung für Urologie mit ihrer beruflichen Situation der Befragungen 2015 und 2020 im Vergleich

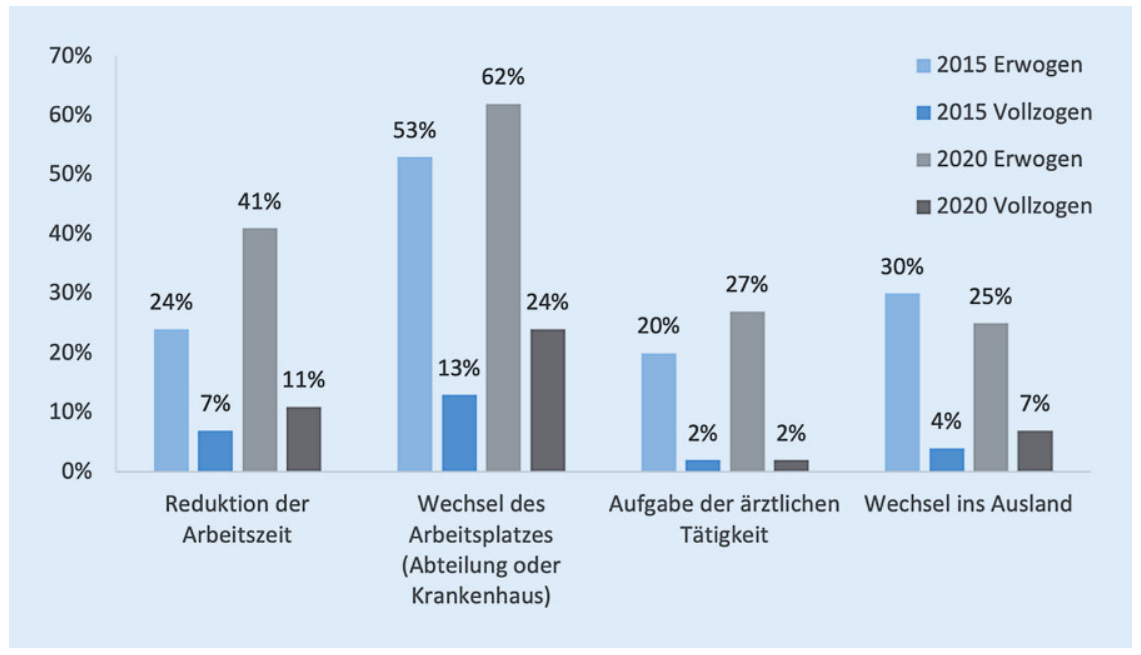

Abb. 2 A Konsequenzen aus Unzufriedenheit über ihre Arbeitsbedingungen der Befragungen 2015 und $2020 \mathrm{im}$ Vergleich (Mehrfachantworten möglich)

mit, innerhalb der Regelweiterbildungszeit von 5 Jahren die vorgeschriebene Weiterbildungsinhalte zu absolvieren. Ein ambulanter Weiterbildungsabschnitt wurde bisher von $3 \%$ absolviert, ist von $6 \%$ geplant und wird von $41 \%$ der Befragten gewünscht.

Jährliche Weiterbildungsgespräche mit dem Weiterbildungsermächtigten finden in $32 \%$ der Fälle in der geforderten Regelmäßigkeit und in $47 \%$ unregelmäßig statt. Hiervon waren nur $26 \%$ der Ansicht, dass die Gespräche für ihre Weiterbildung hilfreich seien. $22 \%$ der Befragten haben keine Weiterbildungsgespräche.

Regelmäßig durchgeführte interne Fortbildungen finden v.a. in einem wöchentlichen $(55 \%)$ oder einem monatli- chen (27\%) Rhythmus statt. $21 \%$ berichten über keine internen Fortbildungsveranstaltungen in ihrer Abteilung. Externe Fortbildungen erachten $48 \%$ als hilfreich und $45 \%$ sogar als unerlässlich, um die Kompetenzen der Weiterbildungsordnung zu erlernen.

\section{Operative Ausbildung}

Die kumulative Anzahl der selbstständig durchgeführten Operationen differenziert nach Weiterbildungsjahr (WBJ) ist in - Abb. 3 dargestellt. Im fünften Weiterbildungsjahr liegt die mediane kumulative Anzahl der selbstständig durchgeführten Operationen bei 102 Eingriffen (1. Quartil 53, 3. Quartil 189).
Bei $45 \%$ der Befragten werden Eingriffe bescheinigt, ohne sie durchgeführt zu haben. Lediglich $28 \%$ führen alle laut WBO geforderten Eingriffe selbst durch. 27\% erfüllen den Operationskatalog nicht vollständig. $64 \%$ der befragten ÄiW sind unzufrieden mit der Art und Häufigkeit ihrer operativen Einsätze.

\section{Vereinbarkeit von Beruf und Familie}

Dass in ihrer Abteilung keine Maßnahmen ergriffen werden, um die Vereinbarkeit von Familie und Beruf zu unterstützen, geben $38 \%$ an. Bei $44 \%$ erfolgt eine Unterstützung durch individuelle Arbeitszeitmodelle, bei $25 \%$ durch Kinderbetreuung. Gesonderte Sozialleistungen wie finanzielle Unterstützung und Notfallkinderbetreuung $(5 \%)$ und ein spezielles Schichtsystem (2\%) gibt es nur selten.

KeineStellen mit reduzierter Wochenarbeitszeit könnten $32 \%$ der Teilnehmenden in ihrer Abteilung in Anspruch nehmen. Es zeigen sich hierbei keine signifikanten Unterschiede in Abhängigkeit von der Trägerschaft der Klinik. $61 \%$ der Arbeitgeber bieten eine betriebliche Kinderbetreuung an, die allerdings aufgrund begrenzter Kapazitäten und nicht passender Betreuungszeiten nur in $20 \%$ genutzt wird bzw. werden könnte. Öffentliche Klinikträger bieten signifikant häufiger eine Kinderbetreuung an als Freigemeinnützige. - Tab. 2 gibt einen Überblick über die subjektive Zufriedenheit zur Vereinbarkeit von Familie und Beruf sowie die Aufteilung der Elternzeit.

\section{Arbeiten in der Schwangerschaft}

Während ihrer klinischen Tätigkeit waren bereits $26 \%$ (35/134) der befragten Frauen schwanger, davon haben $57 \%(20 / 35)$ schwanger operiert. Von diesen würden $95 \%(19 / 20)$ in einer weiteren Schwangerschaft wieder operieren wollen. $79 \%$ (78/99) der befragten Frauen, die noch nicht schwanger waren, würden gerne auch schwanger operieren. Operierende Schwangere haben durchschnittlich in der 11. $\pm 4,6$ Schwangerschaftswoche (SSW) ihre Schwangerschaft bekannt gegeben, bis zur 19. $\pm 9,4$ SSW operiert und bis zur 25. $\pm 8,4$ SSW gearbeitet. Die 0 Abb. 4 zeigt die gewünschten Tätigkeiten im Vergleich zu den tatsächlich durchgeführten 


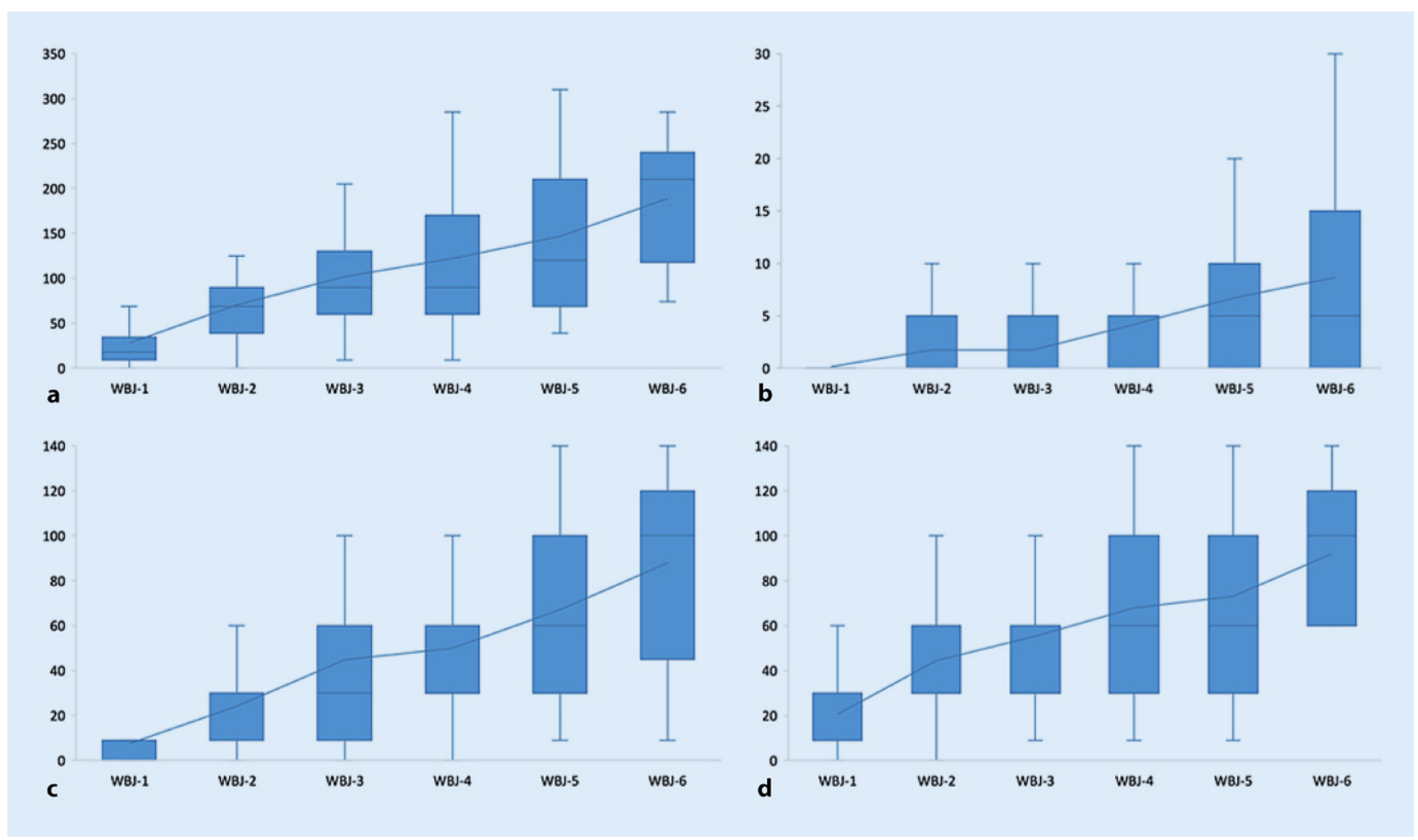

Abb. 3 ॥ Kumulative Anzahl der selbstständig durchgeführten Operationen: a alle Operationen, b kleine Operationen, c endourologische Operationen, $\mathbf{d}$ große Operationen

Tätigkeiten schwangerer ÄiW (Mehrfachauswahl möglich).

\section{Einfluss ökonomischer Erwägungen auf ärztliches Handeln}

Es berichteten $85 \%$ der Befragten, dass ökonomische Erwägungen ihre ärztlichfachlichen Entscheidungen beeinflussen. Bei $35 \%$ war dies selten, bei $31 \%$ jede Woche und bei $20 \%$ beinahe täglich. $69 \%$ werden über die Verweildauer ihrer Patient:innen beispielsweise durch das Controlling oder die Kliniksoftware informiert, davon werden etwa ein Viertel (24\%) sogar durchgehend darauf hingewiesen. Zwei Drittel (66\%) der Befragten haben den Eindruck, dass die Verweildauer den Entlassungszeitpunkt unabhängig von rein medizinisch-fachlichen Erwägungen beeinflusse. Unter einem privaten oder freigemeinnützigen Arbeitgeber wird eine signifikant häufigere Beeinflussung gesehen $(79 \% / 75 \%$ vs. $58 \%$; ANOVA/Tukey; $\left.p=0,009 ; \eta^{2}=0,04\right)$. Arbeitgeber in privater Trägerschaft führen signifikant häufiger DRG-Schulungen durch als Arbeitgeber in öffentlicher oder freigemeinnütziger
Trägerschaft durch (53\% vs. $26 \% / 25 \%$; ANOVA/Tukey; $\left.p=0,001 ; \eta^{2}=0,05\right) .31 \%$ werden durch ein „case management ${ }^{\prime}$ bei der Stationsarbeit unterstützt.

Urologische ÄiW fühlen sich durch den wirtschaftlichen Druck in der Klinik signifikant häufiger belastet, wenn sie bei einer privaten Trägerschaft im Vergleich zu einer öffentlichen Trägerschaft angestellt sind ( $40 \%$ vs. $17 \%$ ). $63 \%$ der Befragten empfinden, dass sich der wirtschaftliche Druck in der Klinik negativ auf ihre Weiterbildung auswirke.

\section{Vereinbarkeit von klinischem Arbeitsalltag mit Forschung und Wissenschaft}

Eine Promotion haben $45 \%$ abgeschlossen. In dieser Umfrage geben $13 \%$ bzw. $71 \%$ an, keine Promotion bzw. Habilitation anzustreben. $46 \%$ der Befragten sind derzeit wissenschaftlich tätig, von denen $71 \%$ unzufrieden mit ihren Forschungsbedingungen sind. Männer sind oder planen signifikant häufiger als Frauen wissenschaftlich tätig zu sein (58\% vs. $35 \%$; t-Test; $95 \%-\mathrm{KI}-0,35$ bis $-0,10 ; p=0,001$; Cohen's $d=0,49)$. Bei der Hälfte der wissenschaftlich Tätigen findet die Forschung außerhalb der regulären klinischen Arbeitszeit statt. Dies stellt mit $80 \%$ den meistgenannten Grund für Unzufriedenheit mit den Forschungsbedingungen dar. Die ÄiW, die nicht wissenschaftlich tätig sein wollen, begründen dies zu $73 \%$ mit fehlender Zeit, zu $50 \%$ mit fehlender Relevanz für ihre Karriere und zu 30\% mit fehlendem wissenschaftlichem Interesse.

\section{Modell beruflicher Gratifikations- krisen}

Die adjustierten Ergebnisse der Erhebung der psychosozialen Arbeitsbelastung nach dem Modell der beruflichen Gratifikationskrisen sind in 0 Tab. 3 dargestellt. Die vorliegende Befragung zeigt eine erhöhte psychosoziale Arbeitsbelastung urologischer ÄiW mit einem Ungleichgewicht von Verausgabungs- und Belohnungsfaktoren (Verausgabungs-Belohnungs-Verhältnis,

ER-Ratio > 1). $86 \%$ der Befragten weisen eine ER-Ratio > 1 auf, $25 \%$ sogar eine ERRatio $>2$. 
Tab. 2 Subjektive Zufriedenheit zum Thema Vereinbarkeit von Beruf und Familie und die Aufteilung der Elternzeit differenziert nach Geschlecht und im Vergleich zu 2015 (in Schulnoten 1-6)

\begin{tabular}{|l|l|l|l|l|l|l|}
\hline & \multicolumn{2}{l|}{$\mathbf{2 0 1 5}$} & \multicolumn{2}{l|}{$\mathbf{2 0 1 5}$} \\
\hline $\begin{array}{l}\text { Wie sind Familie und Beruf bei Ihnen in der Abteilung miteinander zu } \\
\text { vereinbaren? }\end{array}$ & Alle & Frauen & Männer & Alle & Frauen & Männer \\
\hline $\begin{array}{l}\text { Wie werden Mitarbeiter Ihrer Klinik bei der Planung von Elternzeit und dem } \\
\text { Wiedereinstieg unterstützt? }\end{array}$ & $3,3 \pm 1,4$ & $3,7 \pm 1,3$ & $3,6 \pm 1,3$ & $3,7 \pm 1,2$ & $3,8 \pm 1,3$ & $3,5 \pm 1,1$ \\
\hline Wie gestaltet sich der Wiedereinstieg nach der Elternzeit in Ihrer Abteilung? ${ }^{\mathrm{a}}$ & $2,9 \pm 1,3$ & $3,1 \pm 1,3$ & $2,7 \pm 1,3$ & $3,0 \pm 1,3$ & $3,2 \pm 1,3$ & $2,6 \pm 1,2$ \\
\hline $\begin{array}{l}\text { Wie viel Elternzeit haben/würden Sie pro Kind in Anspruch nehmen? (in Mo- } \\
\text { naten) }\end{array}$ & $6,6 \pm 5,2$ & $9,5 \pm 5,1$ & $4,3 \pm 2,9$ & $7,4 \pm 5,2$ & $10,4 \pm 4,7$ & $3,7 \pm 3,0$ \\
\hline${ }^{a}$ Angabe in Schulnoten (1 - sehr gut, 6- ungenügend) & $3,4 \pm 1,3$ & $3,5 \pm 1,4$ & $3,3 \pm 1,4$ \\
\hline
\end{tabular}

${ }^{a}$ Angabe in Schulnoten (1 - sehr gut, 6 - ungenügend)

Befragte mit fortgeschrittener Weiterbildung haben höhere ER-Ratios. Bei Vorliegen eines strukturierten Weiterbildungscurriculums innerhalb des Klinikums zeigt sich eine signifikant geringere ERRatio als in der Vergleichsgruppe (t-Test; $95 \%-\mathrm{KI}-0,74$ bis $-0,20 ; p<0,001$; Cohen's $d=0,33$ ). Je gefährdeter die Befragten die Qualität der Krankenversorgung sehen, desto höher liegt deren ER-Ratio (Antwortmöglichkeiten "Weiß nicht" und "Nein, überhaupt nicht" ausgeschlossen; ANOVA/Tukey; $p<0,001 ; \eta^{2}=0,10$, "Nein, eher nicht“ vs. „Ja, sehr deutlich“: $95 \%-\mathrm{KI}$ $-1,74$ bis $-0,38, p<0,001$; „Ja, eher schon“ vs. „Ja, sehr deutlich“: $95 \%-\mathrm{KI}-1,37$ bis $-0,42, p<0,001$ ).

Signifikante Unterschiede der ER-Ratios liegen zudem bei einer Differenzierung nach Geschlecht (t-Test; 95\%-KI 0,01 bis $0,73 ; p=0,039$; Cohen's $d=0,26)$, nach der wöchentlichen Arbeitszeit (ANOVA/Tukey; $p=0,034 ; \eta^{2}=0,03,<50$ h vs. $\geq 60$ h: $95 \%$ $\mathrm{KI}-1,19$ bis $-0,05, p=0,029)$ und nach der Anzahl der Dienste (Antwortmöglichkeit "0" ausgeschlossen; ANOVA/Tukey; $p=0,025 ; \eta^{2}=0,03,4-6$ vs. $\geq 7: 95 \%-\mathrm{KI}$ $-1,23$ bis $-0,07, p=0,023$ ) vor.

\section{Diskussion}

Ziel dieser Arbeit ist eine aktuelle Erhebung der Arbeits- und Weiterbildungsbedingungen der angehenden Urolog:innen in Deutschland vor der flächendeckenden Anwendung der 2018 novellierten MWBO [5] und des WECU [25]. Zudem erfolgt ein Vergleich mit den Ergebnissen der Vorbefragung von 2015. Ein neuer Schwerpunkt in dieser Arbeit ist das Operieren in der Schwangerschaft. Mit dieser Umfrage sollen Stärken und Konfliktfelder in der urologischen Weiterbildung sowie Verbesserungs- und Entwicklungsmöglichkeiten aufzeigt werden, um Medizinstudierende für das Fach Urologie zu begeistern und den urologischen Nachwuchs bestmöglich aus- und weiterzubilden. Im Vergleich zur Vorbefragung von 2015 zeigt sich eine etwas geringere Rücklaufquote. Gründe hierfür könnten in der während der COVID-19-Pandemie („Coronavirus disease $2019^{\prime \prime}$ ) bestehenden höheren Arbeitsbelastung sowie großen Anzahl an parallel durchgeführten Online-Befragungen liegen. Außerdem wäre es möglich, dass weniger ÄiW als 2015 eine solche Umfrage als sinnvoll erachten bzw. glauben, durch solche Umfragen etwas bewirken zu können und sich deshalb gegen eine Teilnahme entschieden haben.

\section{Ärztliche Weiterbildung}

Bereits 2015 waren die Befragten mit ihrer beruflichen Situation durchaus zufrieden. Diese Zufriedenheit steigerte sich in dieser Umfrage um 10\% auf 54\%. Auch die Wahl des Faches Urologie war für fast $80 \%$ die Richtige. Gleichzeitig denken mehr ÄiW darüber nach, die Arbeitszeit zu reduzieren oder den Arbeitsplatz zu wechseln und setzten diese Überlegungen auch öfter als vor 5 Jahren um (- Abb. 2). Als Gründe für Unzufriedenheit wurden v.a. ein zu hoher Anteil an nichtmedizinischer Tätigkeiten, die Arbeitsverdichtung und eine mangelhafte Weiterbildung genannt. Laut einer Umfrage des Marburger Bundes [17] fühlt sich fast die Hälfte der Ärzt:innen häufig überlastet. $75 \%$ der Befragten werden von ihrer Arbeit so stark in Anspruch genommen, dass dadurch deren Privat- und Familienleben leidet.
Die durchschnittliche wöchentliche Arbeitszeit bei Ärzt:innen in Vollzeit liegt bei $56,5 \mathrm{~h}$ - die gewünschte Wochenarbeitszeit dagegen nur bei $42,3 \mathrm{~h}$. Somit werden $14 \mathrm{~h}$ mehr als gewünscht pro Woche gearbeitet - und wie diese Umfrage zeigt, weder systematisch erfasst noch finanziell honoriert. Wertschätzung durch Vorgesetzte, verlässliche Arbeitszeiten und Entlastung durch IT und medizinische Dokumentare könnten Abhilfe leisten [20, 32]. Hierdurch wird auch die Klinik als Arbeitgeber attraktiver. In der Folge sinkt die Personalfluktuation, es müssen weniger Mitarbeitende eingearbeitet werden und die Weiterbildung kann strukturierter erfolgen.

Die vorliegende Erhebung zeigt, dass nur bei einem Viertel der Befragten eine strukturierte Weiterbildung und bei weniger als einem Drittel die vorgesehenen jährlichen Weiterbildungsgespräche stattfinden. Finden Weiterbildungsgespräche statt, führen davon nur $20 \%$ zu einem subjektiven Mehrwert für die Weiterbildung. Hieraus lassen sich Verbesserungsmaßnahmen ableiten, die mit wenig Aufwand umgesetzt werden können: Geschulte Weiterbildungsbefugte, ein transparentes Weiterbildungscurriculum (z.B. das WECU) und standardisierte, zielorientierte Mitarbeiter:innengespräche sind kostengünstige, einfache und wirksame Werkzeuge, um die Zufriedenheit und die Weiterbildung und damit auch die Versorgungsqualität zu verbessern [13, 19, 22, 33].

\section{Operative Ausbildung}

Obwohl sich der Trend eines nicht vollständig erfüllen Operationskatalog zu 2015 


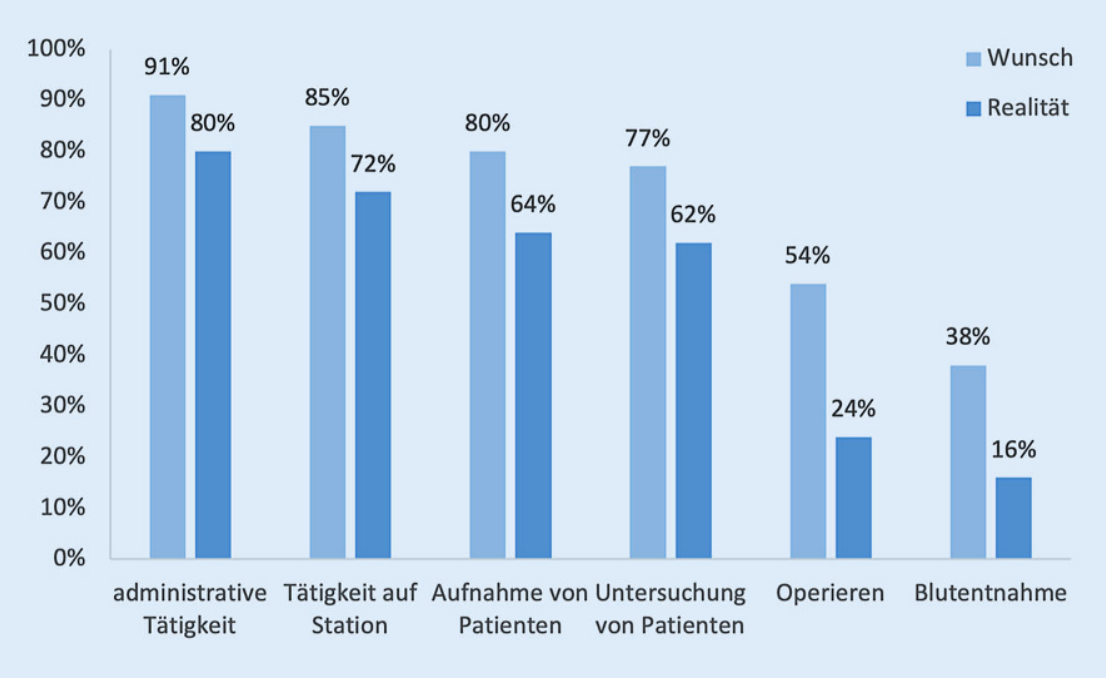

Abb. 4 \ Tätigkeiten schwangerer Ärztinnen in Weiterbildung für Urologie

verstärkt, geben nun die ÄiW an mehr zu operieren. Dies untermauert, dass eine kompetenzbasierte Weiterbildung sinnvoller als eine zahlenorientierte ist und der Realität mehr entspricht. Über $60 \%$ der angehenden Urolog:innen sind wie auch schon vor 5 Jahren unzufrieden mit ihrer operativen Ausbildung. Ebenso viele sind der Meinung, dass die Weiterbildungsoperationen weder transparent noch fair verteilt werden. Ein strukturiertes, transparentes Curriculum stellt auch in der operativen Weiterbildung eine Lösung dar $[5-8,24,27]$. Es sollte einen theoretischen Ausbildungsteil, Simulationstraining und die praktische strukturierte Ausbildung beinhalten. Ein vergleichbarer, einheitlicher Ausbildungsstand der ÄiW erleichtert zudem die Personalplanung bei einem Stellenwechsel.

\section{Vereinbarkeit von Beruf und Familie}

Die Vereinbarkeit von Beruf und Familie, Planung der Elternzeit und der Wiedereinstieg werden konstant, wie auch schon 2015, mit der Schulnote 3 bis 4 bewertet. Eine reduzierte Wochenarbeitszeit scheint individuell weiterhin schwierig umsetzbar zu sein, wird aber vermehrt angeboten (2020: $44 \%$ vs. $2015: 36 \%$ ). Erstaunlich ist, dass im Jahr 2020 noch immer $23 \%$ der Kliniken (2015: 28\%) gar keine Teilzeitmodelle anbieten. Laut der Befragung des Hartmannbundes [15] empfinden $66 \%$ die Arbeitsbelastung als so groß, dass das Privat- und Familienleben darunter leiden weil soziale Kontakte abbrechen und familiäre Zuwendung vernachlässigt wird. Dass der Arbeitgeber keine flexible Arbeitszeitengestaltung ermöglicht wird in derselben Umfrage von $45 \%$ als das größte Hindernis bei der Vereinbarkeit von Familie und Beruf angesehen. Die Autor:innen schließen sich den Empfehlungen des BMSFJF [12] an und fordern die Krankenhäuser als Arbeitgeber auf Vereinbarkeitsangebote zu kommunizieren, flexible Arbeitszeitmodelle zu fördern, Entlastungsangebote und Kinderbetreuung anzubieten und Karrieren nicht an Vollzeittätigkeit zu binden.

\section{Arbeiten in der Schwangerschaft}

Die überwiegende Mehrheit der Ärztinnen in Weiterbildung möchte gerne schwanger operieren [30]. Gefragt nach den Tätigkeiten, welche schwangere ÄiW ausüben dürfen und welche sie tatsächlich ausüben, zeigt sich eine Diskrepanz der Erwartungen und der gelebten Realität ( $\bullet$ Abb. 4). Gut ausgebildeten, in den Klinikablauf integrierten Urologinnen sollte es möglich sein, unter vollem Mutterschutz weiterzuarbeiten. Weitere Arbeiten bestätigen, dass die große Mehrheit der ÄiW auch schwanger operieren will [14, 21, 21, 23]. Auch das Mutterschutzgesetz (MuSchG), zuletzt 2018 novelliert, erlaubt Interventionen. 2021 beleuchteten Arnold et al. erstmals die haftungsrechtlichen Aspekte für schwangere urologisch Tätige [4]. Unter Berücksichtigung der Vorschriften des MuSchG übersteigt das Haftungsrisiko nicht jenes, welches der Arbeitgeber i. Allg. zu tragen hat. Arbeitsverbote gegen den Willen der Schwangeren sollten somit vermieden werden [26].

\section{Einfluss ökonomischer Erwägungen auf ärztliches Handeln}

Die Ökonomisierung ist im Arbeitsalltag der Befragten weiterhin spürbar. Dabei stieg im Vergleich zur Vorbefragung sowohl der Anteil, der den wirtschaftlichen Druck als belastend empfindet ( $45 \%$ vs. $38 \%)$, als auch der Anteil, der negative Auswirkungen auf seine Weiterbildung empfindet ( $63 \%$ vs. $55 \%$ ). Ähnliche Zahlen erheben auch Weiterbildungsumfragen aus anderen Fachrichtungen [18]. Junge Mediziner:innen wünschen sich, dass ökonomische Aspekte medizinisch-fachliche Entscheidungen weniger beeinflussen [32]. Dass konkrete Konzepte wie Forschung und Weiterbildung in der Vergütung der Krankenversorgung berücksichtigt werden können, wäre wünschenswert. Es sollte festgelegt werden, wie ökonomische Erwägungen in der Aus- und Weiterbildung des ärztlichen Nachwuchses geschult werden und wo die Grenzen dieser ökonomischen Erwägungen liegen sollten.

\section{Vereinbarkeit von klinischem Arbeitsalltag mit Forschung und Wissenschaft}

Forschung und Wissenschaft sind für die Befragten weiterhin schwer mit dem klinischen Arbeitsalltag zu vereinbaren. Der Trend zur Promotion und Habilitation geht etwas zurück. $13 \%$ bzw. $71 \%$ geben an, keine Promotion bzw. Habilitation anzustreben; 3\% mehr als 2015. Die generelle Bereitschaft, wissenschaftlich zu arbeiten, steigt jedoch deutlich um $7 \%$ auf $46 \%$. $56 \%$ der Männer geben an, wissenschaftlich zu arbeiten oder dies vorzuhaben (2015: 53\%), jedoch nur 35\% (2015: 29\%) der Frauen. Warum dieser signifikante Unterschied zwischen den Geschlechtern besteht, kann diese Umfrage nicht abschließend beantworten. Die Autor:innen schließen sich dem Maßnahmenkatalog zur urologischen Forschungsförderung von Ralla et al. an, der strukturell ausgebaute Clinical-scientist- 
Tab. 3 Psychosoziale Arbeitsbelastung nach dem Modell der beruflichen Gratifikationskrisen

\begin{tabular}{|c|c|c|c|c|}
\hline & $\begin{array}{l}\text { Verausgabung } \\
(0-100)\end{array}$ & $\begin{array}{l}\text { Belohnung } \\
(0-100)\end{array}$ & ER-Ratio & $\begin{array}{l}\text { Verausgabungs- } \\
\text { neigung }(0-100)\end{array}$ \\
\hline & \multicolumn{4}{|c|}{ Adjustierter Mittelwert \pm Standardabweichung } \\
\hline Alle & $79 \pm 17$ & $53 \pm 15$ & $1,79 \pm 1,45$ & $51 \pm 19$ \\
\hline \multicolumn{5}{|l|}{ Geschlecht } \\
\hline Männlich & $77 \pm 18^{*}$ & $55 \pm 14^{*}$ & $1,59 \pm 1,14^{*}$ & $47 \pm 19^{*}$ \\
\hline Weiblich & $82 \pm 17^{*}$ & $51 \pm 16^{*}$ & $1,97 \pm 1,66^{*}$ & $54 \pm 19^{*}$ \\
\hline \multicolumn{5}{|l|}{ Trägerschaft der Klinik } \\
\hline Öffentlich & $79 \pm 17$ & $54 \pm 15$ & $1,63 \pm 0,73$ & $50 \pm 20$ \\
\hline Freigemeinnützig & $80 \pm 16$ & $53 \pm 15$ & $1,92 \pm 2,05$ & $50 \pm 18$ \\
\hline Privat & $81 \pm 19$ & $49 \pm 18$ & $2,12 \pm 1,76$ & $54 \pm 19$ \\
\hline \multicolumn{5}{|l|}{ Kinder im Haushalt } \\
\hline Ja & $80 \pm 19$ & $49 \pm 14^{*}$ & $2,01 \pm 1,97$ & $51 \pm 18$ \\
\hline Nein & $79 \pm 17$ & $54 \pm 16^{*}$ & $1,71 \pm 1,19$ & $51 \pm 19$ \\
\hline \multicolumn{5}{|l|}{ Forschend } \\
\hline $\mathrm{Ja}$ & $77 \pm 19^{*}$ & $53 \pm 15$ & $1,80 \pm 1,89$ & $51 \pm 18$ \\
\hline Nein & $82 \pm 16^{*}$ & $53 \pm 16$ & $1,78 \pm 0,92$ & $51 \pm 20$ \\
\hline \multicolumn{5}{|l|}{ Weiterbildungsjahr } \\
\hline Jahre 1 und 2 & $76 \pm 16^{*}$ & $58 \pm 14^{*}$ & $1,45 \pm 0,62^{*}$ & $48 \pm 19$ \\
\hline Jahr 3 & $82 \pm 17$ & $53 \pm 17$ & $1,75 \pm 0,81$ & $51 \pm 19$ \\
\hline Jahre 4 und 5 & $82 \pm 17^{*}$ & $50 \pm 15^{*}$ & $2,07 \pm 2,09^{*}$ & $54 \pm 20$ \\
\hline \multicolumn{5}{|c|}{ Wöchentliche Arbeitsstunden } \\
\hline$<50$ & $73 \pm 20^{* 1,3}$ & $54 \pm 14$ & $1,48 \pm 0,65^{* 1}$ & $45 \pm 16^{* 1,2}$ \\
\hline $50-59$ & $80 \pm 15^{* 2,3}$ & $54 \pm 17$ & $1,84 \pm 1,79 * 2$ & $52 \pm 20^{* 2}$ \\
\hline$\geq 60$ & $87 \pm 15^{* 1,2}$ & $49 \pm 14$ & $2,10 \pm 1,45^{* 1,2}$ & $56 \pm 20^{* 1}$ \\
\hline \multicolumn{5}{|l|}{ Anzahl der Dienste } \\
\hline $1-3$ & $80 \pm 20$ & $52 \pm 13$ & $1,66 \pm 0,64$ & $48 \pm 18$ \\
\hline $4-6$ & $80 \pm 17$ & $54 \pm 15^{*}$ & $1,70 \pm 1,19^{*}$ & $50 \pm 19$ \\
\hline$\geq 7$ & $82 \pm 15$ & $48 \pm 17^{*}$ & $2,35 \pm 2,48^{*}$ & $57 \pm 19$ \\
\hline \multicolumn{5}{|c|}{ Strukturiertes Weiterbildungscurriculum } \\
\hline Vorhanden & $77 \pm 17$ & $57 \pm 14^{*}$ & $1,43 \pm 0,52^{*}$ & $46 \pm 17^{*}$ \\
\hline Nicht vorhanden & $80 \pm 17$ & $51 \pm 16^{*}$ & $1,90 \pm 1,61^{*}$ & $52 \pm 19^{*}$ \\
\hline \multicolumn{5}{|l|}{ Weiterbildungsgespräche } \\
\hline Strukturiert & $80 \pm 20$ & $56 \pm 15^{*}$ & $1,63 \pm 1,17$ & $49 \pm 19$ \\
\hline Beiläufig & $80 \pm 16$ & $53 \pm 14$ & $1,73 \pm 1,11$ & $52 \pm 19$ \\
\hline Gar nicht & $78 \pm 16$ & $48 \pm 17^{*}$ & $2,14 \pm 2,19$ & $51 \pm 19$ \\
\hline \multicolumn{5}{|c|}{ Gefahr der Qualität der Patientenversorgung } \\
\hline Nein, eher nicht & $70 \pm 16^{* 1}$ & $56 \pm 13^{* 1}$ & $1,34 \pm 0,48^{* 1}$ & $40 \pm 15^{* 1}$ \\
\hline Ja, eher schon & $77 \pm 16^{* 2}$ & $56 \pm 14^{* 2}$ & $1,50 \pm 0,63^{* 2}$ & $49 \pm 19 * 2$ \\
\hline Ja, sehr deutlich & $87 \pm 15^{* 1,2}$ & $47 \pm 17^{* 1,2}$ & $2,39 \pm 2,12^{* 1,2}$ & $57 \pm 20^{* 1,2}$ \\
\hline \multicolumn{5}{|c|}{ Konsequenzen aus Unzufriedenheit über Arbeitsbedingungen } \\
\hline Reduktion der Arbeitszeit & $80 \pm 21$ & $51 \pm 15$ & $1,69 \pm 0,71$ & $55 \pm 18$ \\
\hline $\begin{array}{l}\text { Wechsel des Arbeitsplat- } \\
\text { zes }\end{array}$ & $81 \pm 20$ & $48 \pm 16$ & $2,11 \pm 1,79$ & $56 \pm 20$ \\
\hline $\begin{array}{l}\text { Aufgabe der ärztlichen } \\
\text { Tätigkeit }\end{array}$ & $92 \pm 11$ & $43 \pm 8$ & $2,21 \pm 0,59$ & $50 \pm 27$ \\
\hline Wechsel ins Ausland & $86 \pm 15$ & $45 \pm 17$ & $2,70 \pm 2,54$ & $58 \pm 21$ \\
\hline Keine Konsequenzen & $79 \pm 17$ & $55 \pm 15$ & $1,67 \pm 1,34$ & $48 \pm 19$ \\
\hline
\end{tabular}

Programme und Forschungsfreistellungen fordert. Des Weiteren sollten die bereits bestehenden Forschungsförderungen aktiver beworben, ein DGU-eigenes Stipendienprogramm etabliert werden [28].

Modell beruflicher Gratifikationskrisen

Im Vergleich zur Vorbefragung zeigt sich insgesamt eine deutlich höhere ER-Ratio einerseits und eine geringere Verausgabung, Belohnung und Verausgabungsneigung andererseits (2015: ER-Ratio $1,37 \pm 0,42$, Verausgabung $83 \pm 14$, Belohnung $64 \pm 12$, Verausgabungsneigung $63 \pm 14)$. Vergleichbare Umfragen aus anderen medizinischen Fachdisziplinen wiesen ebenfalls eine hohe psychosoziale Arbeitsbelastung auf [18]. Dabei liegt die ER-Ratio dieser Befragung im oberen Mittelfeld. Studien belegen, dass eine erhöhte psychosoziale Arbeitsbelastung negativen Einfluss auf die Gesundheit und insbesondere auf ein erhöhtes Burnout-Risiko haben kann [9]. Eine hohe psychosoziale Arbeitsbelastung kann die Behandlungsqualität verschlechtern [20,32]. Auch diese Arbeit bestätigt diesen Zusammenhang: Je ausgeprägter die Gratifikationskrise ist, desto stärker sehen die Befragten die Qualität der Krankenversorgung gefährdet.

\section{Limitationen}

Bei Beurteilung der Studienergebnisse ist die Möglichkeit einer Stichprobenverzerrung zu berücksichtigen. Gegebenenfalls sind mehr unzufriedene ÄiW der Einladung zur Teilnahme gefolgt. Des Weiteren kann nicht ausgeschlossen werden, dass Mitmachende die Befragung mehrfach ausfüllten. Da die Beantwortung relativ lange dauert (ca. $25 \mathrm{~min}$ ) sollte der Anteil vernachlässigbar sein. Es ist möglich, dass neben ÄiW auch Fachärzt:innen sowie ÄiW aus Österreich und der Schweiz an der Umfrage teilnahmen, da die GeSRUMitgliedschaft und der GeSRU-E-Mail-Verteiler nicht an den Weiterbildungsstatus oder Tätigkeitsort gebunden sind. 


\section{Fazit für die Praxis}

- Urologische Ärzt:innen in Weiterbildung sind mit ihrem Beruf zufrieden, äußern aber wie schon 2015 deutliche Kritik an den bestehenden Arbeits- und Weiterbildungsbedingungen.

- Im stark verdichteten Arbeitsalltag fehlt es in der Weiterbildung an Struktur, Transparenz sowie an familien- und forschungsfreundlichen Arbeitsbedingungen.

- Die überwiegende Mehrheit der befragten Ärztinnen in Weiterbildung möchte auch schwanger gerne operieren.

- Subjektiv beeinflussen ökonomische Maßgaben medizinische Entscheidungen.

- Die psychosoziale Arbeitsbelastung ist weiterhin sehr hoch mit negativen Folgen für die Behandlungsqualität, die Gesundheit der Ärzt:innen und die Personalfluktuation.

- Für eine gute ärztliche Weiterbildung sind Zeit, Struktur und Feedback wesentliche Faktoren.

\section{Korrespondenzadresse}

\section{Dr. H. Arnold}

Facharztpraxis, Urologie Dr. Hannah Arnold, Gesundheitszentrum Senden Hauptstraße 11 c, 89250 Senden, Deutschland arnold@urologie-senden.de

\section{Einhaltung ethischer Richtlinien}

Interessenkonflikt. H. Arnold, M. Fassbach, A. Mattigk, V. Zehe, A. Beck, F. Wundrack, L. Bellut, J. König und C. Siech geben an, dass kein Interessenkonflikt besteht.

Für diesen Beitrag wurden von den Autoren keine Studien an Menschen oder Tieren durchgeführt. Für die aufgeführten Studien gelten die jeweils dort angegebenen ethischen Richtlinien.

\section{Literatur}

1. Arnold H (2015) Junge Ärzte wollen forschen [Young doctors do want to do research]. Der Urologe 54(11):1620-1621https://doi.org/10. 1007/s00120-015-3985-y

2. Arnold H (2016) Bündnis JUNGE ÄRZTE: Positionspapier [Bündnis JUNGEÄRZTE: position paper]. Der Urologe 55(6):821-822. https://doi.org/10.1007/ s00120-016-0111-8

3. Arnold H, Meyer CP, Salem J et al (2017) Weiterbildungs- und Arbeitsbedingungen urologischer Assistenzärzte in Deutschland. Urologe 56:1311-1319. https://doi.org/10.1007/s00120017-0495-0

4. Arnold H, Beck A, Mattigk A, Himmler M, Harke N $\mathrm{N}$, von Ostau N, Necknig UH (2021) Schwanger in der Urologie! [Working pregnant in urology!]. Der Urologe 60(6):746-752. https://doi.org/10.1007/ s00120-021-01504-w

Training and work conditions of residents in urology in Germany. Results of the second German Society of Residents in Urology e.V. (GeSRU) residency survey from 2020

Background: Residency is the fundation for high-quality medical care and also for career development of young urologists. In 2015 the GeSRU (German Society of Residents in Urology) carried out the first nationwide survey among young physicians in urology and described the status quo of their residency. This revised follow-up examination draws an updated picture of the training and working conditions of residents in urology and assesses the development.

Methods: In 2020 the GeSRU conducted an online-based survey of all residents in urology; therefore, the 2015 questionnaire was expanded. The model of the professional gratification crisis was used again.

Results: A total of 332 questionnaires were analyzed. Major findings have not changed since 2015. The daily working routine is characterized by high pace and workload and economic considerations. Family- and research-friendly working conditions are largely lacking. 35\% of the respondents draw professional consequences. The psychosocial strain remains very high and conveys a risk for physicians' health and patients' quality of care.

Conclusion: These results demonstrate that there are still systemically immanent burdens for residents in urology, which require adjustments to the working and training conditions. A structured, transparent curriculum for urological residency, remuneration and time for training and models which enable work-life balance should be established.

\section{Keywords}

Urologic residency $\cdot$ Surgical training $\cdot$ Occupational stress $\cdot$ Economic pressure $\cdot$ Effort-reward imbalance

5. Bartmann F (2018) Die Novellierung der Musterweiterbildungsordnung. Urologe 57:898-901. https://doi.org/10.1007/s00120-018-0688-1

6. Beyer B, Boehm K, Borgmann H, Janssen M (2015) Mit den Jungen fängt es an. Urologe 54:259-260. https://doi.org/10.1007/s00120-015-3766-7

7. Borgmann H, Arnold HK, Meyer CP et al (2018) Training, research, and working conditions for urology residents in Germany: a contemporary survey. Eur Urol Focus 4:455-460. https://doi.org/ 10.1016/j.euf.2016.12.001

8. Bründl J, Zengerling F, Borgmann H, Syring I (2014) Prostate surgery within residency programs in urology. Urologe A 53:379-381. https://doi.org/ 10.1007/s00120-014-3424-5

9. Buddeberg-Fischer B, Klaghofer R, Stamm M, Siegrist J, Buddeberg C (2008) Work stress and reduced health in young physicians: prospective evidence from Swiss residents. International Archives of Occupational and Environmental Health 82(1):31-38. https://doi.org/10.1007/ s00420-008-0303-7

10. Buddeberg-Fischer B, Stamm M, Buddeberg $C$ et al (2008) Work stress, health and satisfaction of life in young doctors. Results of a longitudinal study in Switzerland. Dtsch Med Wochenschr 133:2441-2447. https://doi.org/10.1055/s-00281100936

11. Bundesärztekammer (2018) Ärztestatistikzum 31. Dezember 2018

12. Bundesministerium für Familie, Senioren, Frauen und Jugend (2020) In 5 Schritten zu mehr Vereinbarkeit Leitfaden für eine erfolgreiche Fachkräftesicherung im Krankenhaus

13. Cebulla A, Bolenz C, Carrion DM, Bellut L (2019) Urology training in Germany: international com- parison of educational concepts and satisfaction. Urologe A 58:132-138. https://doi.org/10.1007/ s00120-019-0854-0

14. Fritze-Büttner F, Dittmar R, Niethard M (2017) Surgery during pregnancy-Regulations and requirements.Zentralbl Chir 142:575-580. https:// doi.org/10.1055/s-0043-121344

15. Hartmannbund (2019) Die große HB-Assistenzarztumfrage 2018/19

16. Hillienhof A, Osterloh F (2019) Assistenzärzte: Zu wenig Zeit für die Patienten. Dtsch Arztebl Int 116:A-671

17. Institut für Qualitätsmessung und Evaluation $\mathrm{mBH}$ (2019) Ergebnisbericht der Mitgliederbefragung Gesamtauswertung-MB-Monitor 2019

18. Joachim SC, Bitzinger D, Arnold H et al (2020) Die Arbeits- und Weiterbildungssituation junger Ärztinnen und Ärzte in Deutschland eine zusammenfassende Analyse von Befragungsergebnissen aus sechs Fachrichtungen. Gesundheitswesen 82:227-235. https://doi.org/ 10.1055/a-0945-9387

19. Kadmon $M$, Ten Cate $O$, Harendza S, Berberat $P$ (2017) Postgraduate medical education-An increasingly important focus of study and innovation. GMS J Med Educ 34:Doc70. https://doi. org/10.3205/zma001147

20. Klein J, Frie KG, Blum K et al (2010) Berufliche Gratifikationskrisen, Job Strain und Burnout bei chirurgisch tätigen Krankenhausärzten. Psychother Psychosom Med Psychol 60:374-379

21. Knieper C, Ramsauer B, Hancke Ketal (2014) “Pregnant and operating": evaluation of a Germanywide survey among female gynaecologists and surgeons. Geburtshilfe Frauenheilkd 74:875-880. https://doi.org/10.1055/s-0034-1383065 
22. König J, Aeishen S, Cebulla A et al (2019) Qualität statt Quantität verbessert die Weiterbildung. Urologe 58:877-880. https://doi.org/10.1007/ s00120-019-0987-1

23. Mayer KL, Ho HS, Goodnight JE Jr (2001) Childbearing and child care in surgery. Arch Surg 136:649-655. https://doi.org/10.1001/archsurg. 136.6.649

24. Meyer CP, Salem J, Kluth LA et al (2016) The GESRU endo-training-Strategies for the optimization of endourological skills for residents. Urologe 55:253-256. https://doi.org/10.1007/s00120015-0015-z

25. Michel MS, Himmler M, Necknig U et al (2020) Zertifiziertes Weiterbildungscurriculum für die Facharztweiterbildung in Urologie der Deutschen Gesellschaft für Urologie gemäß der Musterweiterbildungsordnung 2018 (Fassung vom 20.09.2019). Urologe 59:135-140. https://doi.org/10.1007/ s00120-020-01367-7

26. MohrR(2020) Juristisches Kurzgutachten: Dasärztliche Arbeiten und Operieren in der Schwangerschaft unter konkreter Bezugnahme auf das Fach Urologie einschließlich etwaiger Haftungsrisiken. https://www.gesru.de/fuer-assistenzaerzte/ fortbildung/kurzgutachten-schwangerschaft/. Zugegriffen: 12. Juli 2021

27. Müller SC, Strunk T, Alken P (2012) Quality and objectifiability of training and advanced training in urology. Urologe A 51:1065-1073. https://doi.org/ 10.1007/s00120-012-2934-2

28. Ralla B, Leyh H, Steffens J et al (2019) Konzepte zur besseren Vereinbarkeit von klinischer und wissenschaftlicher Tätigkeit - ein Thesenpapier der Arbeitsgemeinschaft "Junge Urologen“ der Deutschen Gesellschaft für Urologie. Urologe 58:933-937. https://doi.org/10.1007/s00120019-0996-0

29. Raspe $M$, Müller-Marbach A, Schneider $M$ et al (2016) Work and training conditions of young German physicians in internal medicine. Results of a nationwide survey by young internists from the German Society of Internal Medicine and the German Professional Association of Internists. Dtsch Med Wochenschr 141:202-210. https://doi. org/10.1055/s-0041-109329

30. Necknig UH, Arnold H, von Ostau N (2021) Operieren in der Schwangerschaft - ein Tabu? Aktuelle Urologie 52(03):276-280.https://doi.org/ 10.1055/a-1399-7859

31. Raspe M, Vogelgesang A, Fendel J et al (2018) Work and training conditions of young German physicians in internal medicine-Results of a second nationwide survey by young internists from the German Society of Internal Medicine and the German Professional Association of Internists. Dtsch Med Wochenschr 143:e42-e50. https://doi.org/10.1055/a-0543-4544

32. Raspe $M$, Koch P, Zilezinski M et al (2020) Arbeitsbedingungen und Gesundheitszustand junger Ärzte und professionell Pflegender in deutschen Krankenhäusern. Bundesgesundheitsblatt Gesundheitsforschung Gesundheitsschutz 63:113-121. https://doi.org/10.1007/s00103019-03057-y

33. Sonntag U, Koch A, Bayer G et al (2020) Train the trainer course for general practice trainers in ambulatory care: the Berlin model.GMSJMedEduc 37:Doc28. https://doi.org/10.3205/zma001321

34. Zentralinstitut für die kassenärztliche Versorgung in Deutschland (2019) Zi-Praxis-Panel Jahresbericht 2017

\section{DFG \\ Deutsche \\ Forschungsgemeinschaft \\ DGU \\ für Urologie e.V.}

\section{DGU schreibt Teilnahme an der DFG-Nachwuchsakademie "UroAgeCare" aus}

Die neue DFG-Nachwuchsakademie UroAgeCare rückt das Thema "Alter im Kontext von urologischen Erkrankungen und Therapieoptionen" in den Fokus und verfolgt damit das Ziel, durch eine gesteigerte Antragstellung bei der DFG die Forschungstätigkeiten und die daraus resultierenden wissenschaftlichen Erkenntnisse in diesem Bereich der Urologie zu fördern und zu vermehren. Dabei werden junge urologisch forschende Wissenschaftlerinnen und Wissenschaftler in einem frühen Stadium ihrer Karriere auf ihren ersten DFG-Antrag und eine eigenständige Projektleitung herangeführt.

Die Ausschreibung richtet sich an Forscherinnen und Forscher mit medizinischem oder naturwissenschaftlichem Hintergrund, die kurz vor Abschluss ihrer Promotion stehen oder ihre Promotion vor max. 10 Jahren abgeschlossen haben, einer urologischen Klinik oder einem urologischen Forschungslabor angehören oder mit einer solchen Einrichtung assoziiert sind und ein wissenschaftliches Projekt aus dem Themenbereich „Urological Diseases in an Ageing Society - From Genetics to Medical Care" durchführen wollen.

Die Teilnehmerinnen und Teilnehmer der Nachwuchsakademie werden fachspezifisch durch ausgewiesene Wissenschaftlerinnen und Wissenschaftler betreut und sowohl bei der Planung ihrer Forschungsvorhaben als auch bei der Formulierung ihrer DFG-Erstanträge unterstützt. Ein wesentlicher Meilenstein der Nachwuchsakademie ist dabei ein initialer fünftägiger Workshop, in dem sich bis zu 20 Teilnehmerinnen und Teilnehmer mit den Faculty-Mitgliedern und weiteren Gastreferenten zu einem intensiven fachlichen Austausch rund um die wissenschaftliche Agenda von UroAgeCare und die avisierten Projektvorhaben der Teilnehmenden zusammenfinden. Die Ausformulierung der Antragsentwürfe erfolgt dann in einer nächsten Phase, in der die Mentees individuell durch ihre Mentorinnen und Mentoren betreut und beraten werden. Das erste Förderjahr schließt mit der Einreichung der Erstanträge bei der DFG ab.

In der sich anschließenden einjährigen Projektphase erarbeiten die Teilnehmerinnen und Teilnehmer die Grundlagen für eine zweite DFG-Antragstellung - dieses Mal für das normale DFG-Einzelverfahren.

Voraussetzung für die Teilnahme an der Nachwuchsakademie ist die Einreichung einer Projektskizze für ein Forschungsvorhaben mit Beschreibung von Projektidee, Zielsetzung und Grundkonzeption sowie der geplanten Durchführung. Für die Förderwürdigkeit der später bei der DFG einzureichenden (Erst-)Anträge sind vor allem die innovative Idee des Projektentwurfs sowie deren realistische Umsetzbarkeit von Bedeutung.

Interessierte finden alle Informationen zum Bewerbungsverfahren auf der UroAgeCare-Homepage unter https://uroagecare.de

Die Bewerbungsfrist endet am 31. Oktober 2021 